\title{
Cohomology of rigid curves with semi-stable coverings
}

\author{
Naoki Imai and Takahiro Tsushima \\ Dedicated to Professor Robert Coleman \\ with respect for his achievements
}

\begin{abstract}
We construct a semi-stable formal model of a wide open rigid curve with a semi-stable covering, and study the $\ell$-adic cohomology of the rigid curve. We describe the $\ell$-adic cohomology of the rigid curve using the $\ell$-adic cohomology of the irreducible components of a semi-stable reduction, and homology and cohomology of some graphs. We also prove the functoriality of the description for a finite flat morphism that is compatible with semistable coverings of wide open rigid curves.
\end{abstract}

\section{Introduction}

Let $K$ be a complete discretely valued field with a non-trivial valuation. We assume that the residue field $k$ of $K$ is an algebraic extension of a finite field. We consider a wide open rigid curve $W$ over $K$ with a semi-stable covering. The notion of a semi-stable covering of a wide open rigid curve is due to Coleman and Coleman-McMurdy (cf. [Co, $[\mathrm{CM}]$ ). A semi-stable covering of a wide open rigid curve is an analogue of a semi-stable model of an algebraic curve. In fact, Coleman-McMurdy constructed a semi-stable model from a semi-stable covering of a proper smooth curve (cf. CM, Theorem 2.36]). In this paper, we construct a semi-stable formal model of $W$ from a semi-stable covering of $W$.

Let $\ell$ be a prime number different from the characteristic of $k$. The purpose of this paper is to study the $\ell$-adic cohomology of $W$. Let $\mathscr{W}$ be a semi-stable formal model of $W$ constructed from the semi-stable covering of $W$, and let $\Gamma_{\mathscr{W}}$ be the dual graph of the geometric closed fiber of $\mathscr{W}$. In this paper, we describe the $\ell$-adic cohomology of $W$ using the $\ell$-adic cohomology of the irreducible components of the geometric closed fiber of $\mathscr{W}$, and homology and cohomology of some variants of $\Gamma_{\mathscr{W}}$.

We also study a relative situation. Let $W_{1}$ and $W_{2}$ be wide open rigid curves with semistable coverings. We consider a finite flat morphism $f: W_{1} \rightarrow W_{2}$ that is compatible with the semi-stable coverings. We show that such a morphism extends to a morphism between their formal semi-stable models. The pushforward and the pullback on the $\ell$-adic cohomology by $f$ induce morphisms on the $\ell$-adic cohomology of the irreducible components of the geometric closed fibers, and homology and cohomology of the graphs. The induced morphism on the $\ell$-adic cohomology of the irreducible components of the geometric closed fibers is a usual one. We will describe the morphisms on homology and cohomology of the graphs using the induced morphisms on graphs.

2010 Mathematics Subject Classification. Primary: 11G20; Secondary: 14G22. 
The connected components of Lubin-Tate spaces for $G L_{2}$ are examples of wide open rigid curves. The intention of our research is in the application to the study of group actions on the $\ell$-adic cohomology of Lubin-Tate spaces for $G L_{2}$.

\section{Acknowledgment}

The authors thank Yoichi Mieda for reading a manuscript of this paper and giving a lot of suggestions. They thank also Yuichiro Hoshi and Seidai Yasuda for helpful conversations. They are grateful to a referee for suggestions for improvements.

\section{Notation}

Throughout this paper, we use the following notation. For a field $L$ with a non-trivial nonarchimedean valuation, the ring of integers of $L$ is denoted by $\mathcal{O}_{L}$. For a field $F$, the algebraic closure of $F$ is denoted by $\bar{F}$, and the absolute Galois group of $F$ is denoted by $G_{F}$. For a vector space $V$ over a field $F$, the dual vector space of $V$ over $F$ is denoted by $V^{*}$. For a commutative ring $A$, a commutative $A$-algebra $B$ and a scheme $X$ over $A$, the base change of $X$ to $B$ is denoted by $X_{B}$. For an extension $L_{2}$ over $L_{1}$ of fields with nontrivial non-archimedean complete valuations and a rigid space $X$ over $L_{1}$, the base change of $X$ to $L_{2}$ is denoted by $X_{L_{2}}$.

\section{Semi-stable covering}

In this section, we recall the notion of a semi-stable covering and some related results from [CM].

Let $K$ be a complete discretely valued field with a non-trivial valuation $v$. We assume that the residue field $k$ of $K$ is an algebraic extension of a finite field of characteristic $p$. We normalize the valuation so that $v\left(K^{\times}\right)=\mathbb{Z}$. We put $v(0)=+\infty$ and $|x|=p^{-v(x)}$ for $x \in K$. The maximal ideal of $\mathcal{O}_{K}$ is denoted by $\mathfrak{m}_{K}$. Let $\mathbf{C}$ be the completion of an algebraic closure of $K$. The absolute value $|\cdot|$ on $K$ naturally extends to $|\cdot|$ on $\mathbf{C}$.

For $r \in\left|\mathbf{C}^{\times}\right|$, let $B_{K}[r]$ and $B_{K}(r)$ be the rigid spaces over $K$ whose $\mathbf{C}$-valued points are $\{x \in \mathbf{C}|| x \mid \leq r\}$ and $\{x \in \mathbf{C}|| x \mid<r\}$, which we call a closed disk and an open disk respectively. For $r, s \in\left|\mathbf{C}^{\times}\right|$satisfying $r \leq s$, let $A_{K}[r, s]$ and $A_{K}(r, s)$ be the rigid spaces over $K$ whose $\mathbf{C}$-valued points are $\{x \in \mathbf{C}|r \leq| x \mid \leq s\}$ and $\{x \in \mathbf{C}|r<| x \mid<s\}$, which we call a closed annulus and an open annulus respectively. A closed annulus of the form $A_{K}[r, r]$ for $r \in\left|\mathbf{C}^{\times}\right|$is called a circle. For such $r, s$, we define $A_{K}[r, s)$ and $A_{K}(r, s]$ similarly, which we call semi-open annuli.

Definition 1.1. A wide open rigid curve over $K$ is a one-dimensional smooth geometrically connected rigid space $W$ over $K$ which contains affinoid subdomains $X$ and $Y$ such that

1. $W \backslash X$ is a disjoint union of finitely many open annuli,

2. $X$ is relatively compact in $Y$ over $K$ (cf. [BGR, 9.6.2]),

3. $Y \cap V$ is a semi-open annulus for any connected component $V$ of $W \backslash X$. 
We call $X$ an underlying affinoid of $W$.

Theorem 1.2. $[C M$, Theorem 2.18] Let $W$ be a wide open rigid curve over $K$ with an underlying affinoid $X$. Then $W$ may be completed to a proper algebraic curve $C$ over $K$ by gluing closed disks to the connected components of $W \backslash X$.

Let $X$ be a rigid space over $K$. We write $A(X)$ for $\mathcal{O}_{X}(X)$. We put

$$
\begin{aligned}
|f|_{\text {sup }} & =\sup _{x \in X(\mathbf{C})}|f(x)| \quad \text { for } f \in A(X), \\
A^{\circ}(X) & =\left\{\left.f \in A(X)|| f\right|_{\text {sup }} \leq 1\right\}, \\
A^{\circ \circ}(X) & =\{f \in A(X)|| f(x) \mid<1 \text { for all } x \in X(\mathbf{C})\} .
\end{aligned}
$$

Then $A^{\circ}(X)$ is called the ring of bounded rigid analytic functions on $X$. We consider $A^{\circ}(X)$ as a linearly topologized ring with the ideal of definition $A^{\circ \circ}(X)$. We put

Let $X$ be an affinoid rigid space over $K$. We write $\|f\|_{X}$ instead of $|f|_{\text {sup }}$ for $f \in A(X)$.

$$
\bar{X}=\operatorname{Spec}\left(A^{\circ}(X) / A^{\circ \circ}(X)\right),
$$

which we call the reduction of $X$. The reduction $\bar{X}$ is of finite type over $k$ by BGR, 6.3.4. Corollary 3]. The canonical reduction map of $X$ is denoted by $\operatorname{Red}_{X}: X \rightarrow \bar{X}$.

Definition 1.3. A basic wide open pair of rigid curves is a pair $(W, X)$ where $W$ is a wide open rigid curve over $K$ and $X$ is an affinoid subdomain of $W$ such that

1. $\bar{X}$ is an irreducible curve with at worst ordinary double points as singularities,

2. $\|A(X)\|_{X}=|K|$,

3. each connected component of $W \backslash X$ is isomorphic to an annulus of the form $A_{K}(r, 1)$ for $r \in\left|K^{\times}\right|$satisfying $r<1$.

We say that $W$ is a basic wide open rigid curve, if $(W, X)$ is a basic wide open pair for some $X$.

If $(W, X)$ is a basic wide open pair of rigid curves, then $\bar{X}^{\mathrm{c}}$ denotes the compactification of $\bar{X}$ that is smooth at the cusps, where the cusps mean the points in $\bar{X}^{\mathrm{c}} \backslash \bar{X}$.

Definition 1.4. Let $C$ be a wide open rigid curve or a proper smooth curve over K. A semistable covering of $C$ over $K$ is a finite set $\mathcal{S}$ of basic wide open pairs $\left(U, U^{\mathrm{u}}\right)$ such that

1. $\mathcal{S}^{\mathrm{w}}=\left\{U \mid\left(U, U^{\mathrm{u}}\right) \in \mathcal{S}\right\}$ is an admissible covering of $C$,

2. if $U_{1}, U_{2} \in \mathcal{S}^{\mathrm{w}}$ and $U_{1} \neq U_{2}$, then $U_{1} \cap U_{2}$ is a disjoint union of connected components of $U_{1} \backslash U_{1}^{\mathrm{u}}$

3. if $U_{1}, U_{2}$ and $U_{3}$ are three distinct elements of $\mathcal{S}^{\mathrm{w}}$, then $U_{1} \cap U_{2} \cap U_{3}=\emptyset$.

Theorem 1.5. [CM, Theorem 2.36] Let $C$ be a proper smooth curve over $K$. If $C$ has a semistable covering over $K$, then $C$ has an associated semi-stable model over $\mathcal{O}_{K}$ whose reduction has at least two irreducible components.

Remark 1.6. In [CM, Theorem 2.36], it is assumed that $K$ satisfies Hypothesis $T$, which means that $\mathbf{C}$ is isomorphic to the completion of an algebraic closure of a non-archimedean local field. The field $K$ in this paper satisfies Hypothesis T. 


\section{Morphism between graphs}

In this paper, a graph means a finite directed graph such that each edge has two directions.

Let $\Gamma$ be a graph. The set of the vertices of $\Gamma$ is denoted by $\mathcal{V}(\Gamma)$, and the set of the directed edges of $\Gamma$ is denoted by $\mathcal{E}(\Gamma)$. For $e \in \mathcal{E}(\Gamma)$, the source of $e$ and the target of $e$ are denoted by $s(e)$ and $t(e)$ respectively. For $e \in \mathcal{E}(\Gamma)$, let $\bar{e}$ be the directed edge obtained by reversing the direction of $e$.

We take a prime number $\ell$ that is different from $p$. Let $V\left(\Gamma, \mathbb{Q}_{\ell}\right)$ be the $\mathbb{Q}_{\ell}$-vector space generated by $\mathcal{V}(\Gamma)$, and let $E\left(\Gamma, \mathbb{Q}_{\ell}\right)$ be the $\mathbb{Q}_{\ell}$-vector space generated by $\mathcal{E}(\Gamma)$ with relation $e=-\bar{e}$ for all $e \in \mathcal{E}(\Gamma)$. We consider two $\mathbb{Q}_{\ell}$-linear maps

$$
\begin{aligned}
& d: E\left(\Gamma, \mathbb{Q}_{\ell}\right) \longrightarrow V\left(\Gamma, \mathbb{Q}_{\ell}\right) ; e \mapsto t(e)-s(e), \\
& \delta: V\left(\Gamma, \mathbb{Q}_{\ell}\right) \longrightarrow E\left(\Gamma, \mathbb{Q}_{\ell}\right) ; v \mapsto \sum_{e \in \mathcal{E}(\Gamma), t(e)=v} e .
\end{aligned}
$$

We put $H_{1}\left(\Gamma, \mathbb{Q}_{\ell}\right)=\operatorname{Ker}(d)$ and $H^{1}\left(\Gamma, \mathbb{Q}_{\ell}\right)=\operatorname{Coker}(\delta)$. A cycle $R$ in $\Gamma$ can be considered as an element of $H_{1}\left(\Gamma, \mathbb{Q}_{\ell}\right)$. We consider a natural bilinear pairing

$$
\langle,\rangle: E\left(\Gamma, \mathbb{Q}_{\ell}\right) \times E\left(\Gamma, \mathbb{Q}_{\ell}\right) \longrightarrow \mathbb{Q}_{\ell}
$$

determined by

$$
\left\langle e_{1}, e_{2}\right\rangle= \begin{cases}1 & \text { if } e_{1}=e_{2} \\ 0 & \text { if } e_{1} \neq e_{2}, \bar{e}_{2}\end{cases}
$$

for $e_{1}, e_{2} \in \mathcal{E}(\Gamma)$. Then this pairing induces a bilinear perfect pairing

$$
H_{1}\left(\Gamma, \mathbb{Q}_{\ell}\right) \times H^{1}\left(\Gamma, \mathbb{Q}_{\ell}\right) \longrightarrow \mathbb{Q}_{\ell} .
$$

Therefore we have a canonical isomorphism $H^{1}\left(\Gamma, \mathbb{Q}_{\ell}\right) \cong H_{1}\left(\Gamma, \mathbb{Q}_{\ell}\right)^{*}$.

Let $\Gamma_{1}$ and $\Gamma_{2}$ be graphs.

Definition 2.1. A finite flat morphism $\phi: \Gamma_{1} \rightarrow \Gamma_{2}$ of degree $n$ consists of the following data:

- Surjective maps $\phi_{V}: \mathcal{V}\left(\Gamma_{1}\right) \rightarrow \mathcal{V}\left(\Gamma_{2}\right)$ and $\phi_{E}: \mathcal{E}\left(\Gamma_{1}\right) \rightarrow \mathcal{E}\left(\Gamma_{2}\right)$ such that $\phi_{V} \circ s=s \circ \phi_{E}$, $\phi_{E}(\bar{e})=\overline{\phi_{E}(e)}$ for $e \in \mathcal{E}\left(\Gamma_{1}\right)$, and the map $s: \phi_{E}^{-1}\left(e^{\prime}\right) \rightarrow \phi_{V}^{-1}\left(s\left(e^{\prime}\right)\right)$ is surjective for $e^{\prime} \in \mathcal{E}\left(\Gamma_{2}\right)$.

- Positive integers $n_{v}$ and $n_{e}$ for all $v \in \mathcal{V}\left(\Gamma_{1}\right)$ and $e \in \mathcal{E}\left(\Gamma_{1}\right)$ such that $n_{e}=n_{\bar{e}}$ for $e \in \mathcal{E}\left(\Gamma_{1}\right)$,

$$
\sum_{e \in \phi_{E}^{-1}\left(e^{\prime}\right)} n_{e}=n \quad \text { and } \sum_{e \in \phi_{E}^{-1}\left(e^{\prime}\right), s(e)=v} n_{e}=n_{v}
$$

for $e^{\prime} \in \mathcal{E}\left(\Gamma_{2}\right)$ and $v \in \phi_{V}^{-1}\left(s\left(e^{\prime}\right)\right)$.

Let $\phi: \Gamma_{1} \rightarrow \Gamma_{2}$ be a finite flat morphism of degree $n$. For a cycle $R=e_{1} \cdots e_{m}$ in $\Gamma_{1}$, the cycle $\phi_{E}\left(e_{1}\right) \cdots \phi_{E}\left(e_{m}\right)$ in $\Gamma_{2}$ is denoted by $\phi(R)$.

Proposition 2.2. Let $R^{\prime}$ be a cycle in $\Gamma_{2}$. Then there are cycles $R_{1}, \ldots, R_{m}$ in $\Gamma_{1}$ such that $\phi\left(R_{i}\right)=R^{\prime}$ for $1 \leq i \leq m$ and

$$
\left|\left\{1 \leq i \leq m \mid e \in \mathcal{E}\left(R_{i}\right)\right\}\right|=n_{e}
$$

for all $e \in \mathcal{E}\left(\Gamma_{1}\right)$ satisfying $\phi_{E}(e) \in \mathcal{E}\left(R^{\prime}\right)$. Furthermore, $\sum_{i=1}^{m} R_{i} \in H_{1}\left(\Gamma_{1}, \mathbb{Q}_{\ell}\right)$ does not depend on a choice of $R_{1}, \ldots, R_{m}$. 
Proof. By replacing $\Gamma_{1}$ and $\Gamma_{2}$ by their subgraphs, we may assume $\Gamma_{2}=R^{\prime}$.

We prove the first claim by induction on $n$. If $n=1$, the claim is trivial. We assume $n \geq 2$. By the surjectivity of $s: \phi_{E}^{-1}\left(e^{\prime}\right) \rightarrow \phi_{V}^{-1}\left(s\left(e^{\prime}\right)\right)$ for $e^{\prime} \in \mathcal{E}\left(\Gamma_{2}\right)$, we can easily find a cycle $R$ in $\Gamma_{1}$ such that $\phi(R)=R^{\prime}$. Then we put

$$
n_{v}^{\prime}=\left\{\begin{array}{ll}
n_{v}-1 & \text { if } v \in \mathcal{V}(R) \\
n_{v} & \text { if } v \notin \mathcal{V}(R)
\end{array} \quad \text { and } \quad n_{e}^{\prime}= \begin{cases}n_{e}-1 & \text { if } e \in \mathcal{E}(R) \\
n_{e} & \text { if } e \notin \mathcal{E}(R)\end{cases}\right.
$$

for $v \in \mathcal{V}\left(\Gamma_{1}\right)$ and $e \in \mathcal{E}\left(\Gamma_{1}\right)$. We consider the subgraph $\Gamma_{1}^{\prime}$ of $\Gamma_{1}$ obtained from $\Gamma_{1}$ by removing $v \in \mathcal{V}\left(\Gamma_{1}\right)$ and $e \in \mathcal{E}\left(\Gamma_{1}\right)$ such that $n_{v}^{\prime}=0$ and $n_{e}^{\prime}=0$. We define a positive integer $\operatorname{deg}\left(R / R^{\prime}\right)$ by

$$
\phi(R)=\operatorname{deg}\left(R / R^{\prime}\right) R^{\prime} \in H_{1}\left(\Gamma_{2}, \mathbb{Q}_{\ell}\right) .
$$

Then the restriction of $\phi$ gives a finite flat morphism $\phi^{\prime}: \Gamma_{1}^{\prime} \rightarrow \Gamma_{2}$ of degree $n-\operatorname{deg}\left(R / R^{\prime}\right)$ and it suffices to show the claim for $\phi^{\prime}$. This follows from the induction hypothesis.

The last claim follows from the condition $\left|\left\{1 \leq i \leq m \mid e \in \mathcal{E}\left(R_{i}\right)\right\}\right|=n_{e}$ for all $e \in$ $\mathcal{E}\left(\Gamma_{1}\right)$.

We define $\mathbb{Q}_{\ell}$-linear maps $\phi_{*}: H_{1}\left(\Gamma_{1}, \mathbb{Q}_{\ell}\right) \rightarrow H_{1}\left(\Gamma_{2}, \mathbb{Q}_{\ell}\right)$ and $\phi^{*}: H_{1}\left(\Gamma_{2}, \mathbb{Q}_{\ell}\right) \rightarrow H_{1}\left(\Gamma_{1}, \mathbb{Q}_{\ell}\right)$ by $\phi_{*}(R)=\phi(R)$ for a cycle $R$ in $\Gamma_{1}$ and $\phi^{*}\left(R^{\prime}\right)=\sum_{i=1}^{m} R_{i}$ for a cycle $R^{\prime}$ in $\Gamma_{2}$, where $R_{1}, \ldots, R_{m}$ are as in Proposition 2.2.

We define $\phi_{*}: H^{1}\left(\Gamma_{1}, \mathbb{Q}_{\ell}\right) \rightarrow H^{1}\left(\Gamma_{2}, \mathbb{Q}_{\ell}\right)$ and $\phi^{*}: H^{1}\left(\Gamma_{2}, \mathbb{Q}_{\ell}\right) \rightarrow H^{1}\left(\Gamma_{1}, \mathbb{Q}_{\ell}\right)$ as the dual $\mathbb{Q}_{\ell^{-}}$ linear maps of $\phi^{*}: H_{1}\left(\Gamma_{2}, \mathbb{Q}_{\ell}\right) \rightarrow H_{1}\left(\Gamma_{1}, \mathbb{Q}_{\ell}\right)$ and $\phi_{*}: H_{1}\left(\Gamma_{1}, \mathbb{Q}_{\ell}\right) \rightarrow H_{1}\left(\Gamma_{2}, \mathbb{Q}_{\ell}\right)$ respectively.

\section{Formal model of rigid curve}

Let $W$ be a wide open rigid curve with a semi-stable covering $\mathcal{S}=\left\{\left(U_{i}, U_{i}^{\mathrm{u}}\right) \mid i \in I\right\}$ over $K$. In this section, we construct a formal model of $W$ from the semi-stable covering $\mathcal{S}$. First, we recall some facts from $\mathrm{CM}$.

Proposition 3.1. CM, Proposition 2.21] Let $C$ be a proper smooth curve over $K$. Let $L$ be a finite Galois extension of $K$, and let $T$ be a finite nonempty Galois stable subset of $C(L)$. Suppose that $\left\{D_{t} \mid t \in T\right\}$ is a Galois stable collection of disjoint open disks over $L$ in $C$ such that $D_{t} \cap T=\{t\}$ for all $t \in T$. Then $C \backslash\left(\bigcup_{t \in T} D_{t}\right)$ is a one-dimensional affinoid over $K$.

Lemma 3.2. CM, Lemma 2.24] Let $f: X \rightarrow Y$ be a morphism between smooth one-dimensional affinoid rigid spaces over $K$. We assume that $\bar{X}$ is irreducible.

(i) If $\bar{f}: \bar{X} \rightarrow \bar{Y}$ is a surjection, then $f$ is a surjection.

(ii) If $\bar{f}(\bar{X}) \subset \bar{Y}$ is an open affine subset and $f: X(\mathbf{C}) \rightarrow Y(\mathbf{C})$ is an injection, then $\bar{f}$ is an injection.

Lemma 3.3. $C M$, Proposition 2.8] Let $X$ be a smooth one-dimensional affinoid rigid curve such that $\|A(X)\|_{X}=|K|$. We assume that $P \in \bar{X}(\bar{k})$ and $\operatorname{Red}_{X}^{-1}(P)$ is isomorphic to an open annulus over $K$. Then $P$ is an ordinary double point of $\bar{X}$.

For $i \in I$, let $\left\{V_{j} \mid j \in J_{i}\right\}$ be the set of the connected components of $U_{i} \backslash\left(U_{i}^{\mathrm{u}} \cup \bigcup_{i^{\prime} \in I, i^{\prime} \neq i} U_{i^{\prime}}\right)$. For different $i_{1}, i_{2} \in I$, let $\left\{V_{j} \mid j \in J_{i_{1}, i_{2}}\right\}$ be the set of the connected components of $U_{i_{1}} \cap U_{i_{2}}$. 
For $i \in I$ and $j \in J_{i}$, let $V_{j}^{\mathrm{s}}$ be the semi-open annulus obtained by adding a circle $C_{j}$ to $V_{j}$ from the opposite side to $U_{i}^{\mathrm{u}}$. We define a rigid space $W^{\prime}$ as $W \cup \bigcup_{i \in I, j \in J_{i}} V_{j}^{\mathrm{s}}$. We put $\widetilde{I}=I \cup \bigcup_{i \in I} J_{i}$, and

$$
\left(U_{i}, U_{i}^{\mathrm{u}}\right)= \begin{cases}\left(U_{i}, U_{i}^{\mathrm{u}}\right) & \text { if } i \in I \\ \left(V_{i}^{\mathrm{s}}, C_{i}\right) & \text { if } i^{\prime} \in I \text { and } i \in J_{i^{\prime}}\end{cases}
$$

for $i \in \widetilde{I}$. We put

$$
J_{i_{1}, i_{2}}= \begin{cases}J_{i_{1}, i_{2}} & \text { if } i_{1}, i_{2} \in I \\ \left\{i_{1}\right\} & \text { if } i_{2} \in I \text { and } i_{1} \in J_{i_{2}} \\ \left\{i_{2}\right\} & \text { if } i_{1} \in I \text { and } i_{2} \in J_{i_{1}} \\ \emptyset & \text { otherwise }\end{cases}
$$

for different $i_{1}, i_{2} \in \widetilde{I}$.

Proposition 3.4. For $i \in \widetilde{I}$, let $S_{i}$ be a nonempty $G_{k}$-stable collection of points of the smooth locus of $\overline{U_{i}^{\mathrm{u}}}$. We put $U\left(S_{i}\right)=U_{i}^{\mathrm{u}} \backslash\left(\bigcup_{s \in S_{i}} \operatorname{Red}_{U_{i}^{\mathrm{u}}}^{-1}(s)\right)$ for $i \in \widetilde{I}$ and $X_{j}\left(S_{i_{1}}, S_{i_{2}}\right)=U\left(S_{i_{1}}\right) \cup$ $U\left(S_{i_{2}}\right) \cup V_{j}$ for different $i_{1}, i_{2} \in \widetilde{I}$ and $j \in J_{i_{1}, i_{2}}$. Then $U\left(S_{i}\right)$ and $X_{j}\left(S_{i_{1}}, S_{i_{2}}\right)$ are affinoid rigid spaces over $K$, and $\operatorname{Red}_{X_{j}\left(S_{i_{1}}, S_{i_{2}}\right)}$ is compatible with $\operatorname{Red}_{U\left(S_{i_{1}}\right)}$ and $\operatorname{Red}_{U\left(S_{i_{2}}\right)}$ for $i \in \widetilde{I}$ and different $i_{1}, i_{2} \in \widetilde{I}$ and $j \in J_{i_{1}, i_{2}}$.

Proof. We put $W^{\prime}\left(\left\{S_{i}\right\}_{i \in \tilde{I}}\right)=W^{\prime} \backslash\left(\bigcup_{s \in S_{i}, i \in \tilde{I}} \operatorname{Red}_{U_{i}^{\mathrm{u}}}^{-1}(s)\right)$. Let $C$ be a proper smooth curve over $K$ obtained from $W$ as in Theorem [1.2, Then $W^{\prime}\left(\left\{S_{i}\right\}_{i \in \widetilde{I}}\right)$ is obtained from $C$ removing a $G_{K^{-}}$-stable union of disjoint open disks. Therefore $W^{\prime}\left(\left\{S_{i}\right\}_{i \in \widetilde{I}}\right)$ is an affinoid rigid space over $K$ by Proposition 3.1 .

We take $i \in \widetilde{I}$. We know that $U\left(S_{i}\right)$ is an affinoid rigid space by $\mathrm{FvdP}$, Lemma 4.8.1.(a)]. The natural inclusion map $j_{U\left(S_{i}\right)}: U\left(S_{i}\right) \rightarrow W^{\prime}\left(\left\{S_{i}\right\}_{i \in \widetilde{I}}\right)$ induces a map on the reduction $\bar{j}_{U\left(S_{i}\right)}: \bar{U}\left(S_{i}\right) \rightarrow \overline{W^{\prime}}\left(\left\{S_{i}\right\}_{i \in \tilde{I}}\right)$. Let $\operatorname{Im}\left(\bar{j}_{U\left(S_{i}\right)}\right)$ be the image of $\bar{j}_{U\left(S_{i}\right)}$. Then $\operatorname{Im}\left(\bar{j}_{U\left(S_{i}\right)}\right)$ is a point or an affine open subset of $\overline{W^{\prime}}\left(\left\{S_{i}\right\}_{i \in \widetilde{I}}\right)$.

We assume that $\operatorname{Im}\left(\bar{j}_{U\left(S_{i}\right)}\right)$ is a point $P$. Then $\operatorname{Red}_{W^{\prime}\left(\left\{S_{i}\right\}_{i \in \tilde{I}}\right)}^{-1}(P)$ is not connected to $\bigcup_{s \in S_{i}} \operatorname{Red}_{U_{i}^{\mathrm{u}}}^{-1}(s)$. Therefore $U\left(S_{i}\right) \subset \operatorname{Red}_{W^{\prime}\left(\left\{S_{i}\right\}_{i \in \tilde{I}}\right)}^{-1}(P)$ is not connected to $\bigcup_{s \in S_{i}} \operatorname{Red}_{U_{i}^{u}}^{-1}(s)$. This is a contradiction. Thus we have proved that $\operatorname{Im}\left(\bar{j}_{U\left(S_{i}\right)}\right)$ is an affine open subset of $\overline{W^{\prime}}\left(\left\{S_{i}\right\}_{i \in \widetilde{I}}\right)$. Then the map $\bar{j}_{U\left(S_{i}\right)}$ is an injection by Lemma 3.2 (ii). Further, we have that $U\left(S_{i}\right)=\operatorname{Red}_{W^{\prime}\left(\left\{S_{i}\right\}_{i \in \tilde{I}}\right)}^{-1}\left(\operatorname{Im}\left(\bar{j}_{U\left(S_{i}\right)}\right)\right)$ by Lemma $3.2(\mathrm{i})$. Let $Y\left(S_{i}\right)$ be the irreducible component of $\overline{W^{\prime}}\left(\left\{S_{i}\right\}_{i \in \tilde{I}}\right)$ that contains $\operatorname{Im}\left(\bar{j}_{U\left(S_{i}\right)}\right)$.

We take $j \in J_{i_{1}, i_{2}}$ for different $i_{1}, i_{2} \in \widetilde{I}$. Then $V_{j}=\operatorname{Red}_{W^{\prime}\left(\left\{S_{i}\right\}_{i \in \tilde{I}}\right)}^{-1}\left(y_{j}\right)$ for a point $y_{j}$ of $\overline{W^{\prime}}\left(\left\{S_{i}\right\}_{i \in \widetilde{I}}\right)$ by the connectedness of $V_{j}$. Further, we have that $y_{j} \in Y\left(S_{i_{1}}\right) \cap Y\left(S_{i_{2}}\right)$, because $V_{j}$ is connected to $U\left(S_{i_{1}}\right)$ and $U\left(S_{i_{2}}\right)$.

Therefore we have

$$
Y\left(S_{i}\right)=\operatorname{Im}\left(\bar{j}_{U\left(S_{i}\right)}\right) \cup \bigcup_{j \in J_{i, i^{\prime}}, i^{\prime} \in \widetilde{I}}\left\{y_{j}\right\}
$$

for all $i \in \widetilde{I}$. Then $X_{j}\left(S_{i_{1}}, S_{i_{2}}\right)=\operatorname{Red}_{W^{\prime}\left(\left\{S_{i}\right\}_{i \in \tilde{I}}\right)}^{-1}\left(\operatorname{Im}\left(\bar{j}_{U\left(S_{i_{1}}\right)}\right) \cup \operatorname{Im}\left(\bar{j}_{U\left(S_{i_{2}}\right)}\right) \cup\left\{y_{j}\right\}\right)$ is an affinoid rigid space by $[\mathrm{FvdP}$, Lemma 4.8.1.(a)]. The compatibility also follows from [FvdP, Lemma 4.8.1.(c)]. 
For a formal scheme $\mathscr{X}$ over $\operatorname{Spf} \mathcal{O}_{K}$, the closed fiber $\mathscr{X}_{k}$ of $\mathscr{X}$ means the underlying reduced scheme of the ringed space $\left(\mathscr{X}, \mathcal{O}_{\mathscr{X}} / \mathcal{I}\right)$, where $\mathcal{I}$ is an ideal of definition of $\mathscr{X}$.

Theorem 3.5. Let $W$ be a wide open rigid curve with a semi-stable covering $\mathcal{S}=\left\{\left(U_{i}, U_{i}^{\mathrm{u}}\right) \mid\right.$ $i \in I\}$ over $K$. Then $W$ has an associated semi-stable formal model over $\mathcal{O}_{K}$.

Proof. We take $S_{i}$ for $i \in \widetilde{I}$ as in Proposition 3.4. Then $\left\{U_{i}^{\mathrm{u}}\right\}_{i \in \widetilde{I}} \cup\left\{X_{j}\left(S_{i_{1}}, S_{i_{2}}\right)\right\}_{i_{1}, i_{2} \in \widetilde{I}, j \in J_{i_{1}, i_{2}}}$ is a pure affinoid covering of $W^{\prime}$ in the sense of [FvdP, Definition 4.8.3] by Lemma 3.4] This covering gives a formal model $\mathscr{W}^{\prime}$ of $W^{\prime}$. The closed fiber $\mathscr{W}_{k}^{\prime}$ is a semi-stable curve by Lemma 3.3 .

Let $Y$ be the reduced closed subscheme of $\mathscr{W}_{k}^{\prime}$ determined by $\bigcup_{i \in I}{\overline{U_{i}^{\mathrm{u}}}}^{\mathrm{c}}$. We define $\mathscr{W}$ as the formal completion of $\mathscr{W}^{\prime}$ along $Y$. Then $\mathscr{W}$ is a formal model of $W$. We can check that $\mathscr{W}$ is independent of a choice of $S_{i}$ for $i \in \widetilde{I}$.

We use the notation in the proof of Theorem 3.5. The closed fiber $\mathscr{W}_{k}$ of $\mathscr{W}$ is $Y$. The irreducible components of the geometric closed fiber $\mathscr{W}^{\prime}{ }_{\bar{k}}$ of $\mathscr{W}^{\prime}$ consists of proper curves that are also irreducible components of $Y_{\bar{k}}$ and affine lines over $\bar{k}$.

Proposition 3.6. For $i \in I$ and $j \in J_{i}$, we put $X_{j}^{\prime}\left(S_{i}\right)=U\left(S_{i}\right) \cup V_{j}$. Then $\mathscr{W}$ is obtained also by patching $\operatorname{Spf} A^{\circ}\left(U_{i}^{\mathrm{u}}\right)$ for $i \in I$, $\operatorname{Spf} A^{\circ}\left(X_{j}\left(S_{i_{1}}, S_{i_{2}}\right)\right)$ for $i_{1}, i_{2} \in I$ and $j \in J_{i_{1}, i_{2}}$, and $\operatorname{Spf} A^{\circ}\left(X_{j}^{\prime}\left(S_{i}\right)\right)$ for $i \in I$ and $j \in J_{i}$.

Proof. We need to show that the ring of an affine open formal subscheme of $\mathscr{W}$ can be recovered as the ring of bounded rigid analytic functions on its rigid generic fiber. This follows from deJ, Theorem 7.4.1].

Let $C$ be the proper smooth curve over $K$ obtained from $W$ as in Theorem 1.2 . Let $\left\{D_{i^{\prime}}\right\}_{i^{\prime} \in I^{\prime}}$ be the set of the connected components of $D=C \backslash W$. For $i^{\prime} \in I^{\prime}$, there uniquely exist $i \in I$ and $j \in J_{i}$ such that the union of $D_{i^{\prime}}$ and $V_{j}$ defines an open disk in $C$, which is denoted by $U_{i^{\prime}}$. Then $\mathcal{S}^{\prime}=\mathcal{S} \cup\left\{\left(U_{i^{\prime}}, D_{i^{\prime}}\right) \mid i^{\prime} \in I^{\prime}\right\}$ is a semi-stable covering of $C$. Let $\mathcal{C}$ be the semi-stable model of $C$ associated to the semi-stable covering $\mathcal{S}^{\prime}$ by Theorem 1.5. Then $Y$ is naturally considered as a closed subscheme of the special fiber $\mathcal{C}_{k}$ of $\mathcal{C}$.

Proposition 3.7. The formal completion of $\mathcal{C}$ along $Y$ is naturally isomorphic to $\mathscr{W}$.

Proof. This follows from the construction of $\mathscr{W}$ in the proof of Theorem 3.5 .

Remark 3.8. Proposition 3.6 and Proposition 3.7 give two different descriptions of the same formal model $\mathscr{W}$, and the both descriptions are important. The construction in Proposition 3.6 implies that a finite flat morphism between wide open rigid curves which is compatible with their semi-stable coverings naturally extends to a morphism between their semi-stable formal models. This fact is very non-trivial from the construction in Proposition 3.7, because such a morphism does not extend to a morphism between their compactifications in general. On the other hand, by Proposition [3.7, we see that $W$ satisfies the condition of [Fa, Proposition 5.9.4].

\section{Cohomology of rigid curve}

We put $\mathscr{W}_{\mathcal{O}_{\mathbf{C}}}=\mathscr{W} \widehat{\otimes}_{\mathcal{O}_{K}} \mathcal{O}_{\mathbf{C}}$. The formal nearby cycle functor $R \Psi_{\mathscr{W}_{\mathcal{O}_{\mathrm{C}}}}$ of $\mathscr{W}_{\mathcal{O}_{\mathrm{C}}}$ is defined in $\mathrm{Be2}$, section 2]. 
Let $\Gamma_{\mathscr{W}}$ and $\Gamma_{\mathscr{W}}$ be the dual graphs of $\mathscr{W}_{\bar{k}}$ and $\mathscr{W}_{\bar{k}}^{\prime}$ respectively (cf. Li, Definition 10.3.17] ). Then $\Gamma_{\mathscr{W}}$ is a subgraph of $\Gamma_{\mathscr{W}^{\prime}}$. For $v \in \mathcal{V}\left(\Gamma_{\mathscr{W}^{\prime}}\right)$, let $Y_{v}$ be the irreducible component of $\mathscr{W}_{\bar{k}}^{\prime}$ corresponding to $v$, let $\widetilde{Y}_{v}$ be the normalization of $Y_{v}$, and let $\pi_{v}: \widetilde{Y}_{v} \rightarrow \mathscr{W}_{\bar{k}}^{\prime}$ be a natural morphism.

Proposition 4.1. Let $\Lambda$ be a torsion local finite $\mathbb{Z}_{\ell}$-algebra. Then there are canonical isomorphisms

$$
\begin{aligned}
& R^{0} \Psi_{W_{\mathcal{O}_{\mathbf{C}}}} \Lambda \cong \Lambda, \\
& R^{1} \Psi_{\mathscr{W}_{\mathcal{O}_{\mathbf{C}}}} \Lambda \cong\left(\left(\bigoplus_{v \in \mathcal{V}\left(\Gamma_{\mathscr{W}^{\prime}}\right)} \pi_{v *} \Lambda\right) / \Lambda\right)^{*}(-1),
\end{aligned}
$$

where we consider the right hand side of (4.2) as a sheaf on $\mathscr{W}_{\bar{k}}$.

Proof. The isomorphism (4.1) follows from the definition. By [Be2, Theorem 3.1], we have an isomorphism

$$
\left.R^{1} \Psi_{W_{\mathcal{O}_{\mathbf{C}}}} \Lambda \cong\left(R^{1} \Psi_{\mathcal{C}_{\mathcal{O}_{\bar{K}}}} \Lambda\right)\right|_{\overline{\bar{k}}}
$$

Hence it suffices to prove the isomorphism (4.2) locally at singular points of $\mathscr{W}_{\bar{k}}^{\prime}$.

Let $x_{e}$ be the singular point of $\mathscr{W}_{\frac{k}{\prime}}^{\prime}$ corresponding to $e \in \mathcal{E}\left(\Gamma_{\mathscr{W}^{\prime}}\right)$. Then the formal completion $\mathscr{W}_{e}$ of $\mathcal{C}_{\mathcal{O}_{K}}$ at $x_{e}$ is isomorphic to $\operatorname{Spf} \mathcal{O}_{\mathbf{C}}[[S, T]] /(S T-c)$ for some $c \neq 0 \in \mathfrak{m}_{K}$. Further, $\operatorname{Spf} \mathcal{O}_{\mathbf{C}}[[S, T]] /(S T-c)$ is isomorphic to the formal completion of $\mathcal{X}=\operatorname{Spec} \mathcal{O}_{\bar{K}}[S, T] /(S T-c)$ at the point $x_{0}$ of the special fiber defined by $S=T=0$. Note that $\mathcal{X}_{\bar{K}} \cong \mathbb{G}_{m, \bar{K}}$. Then we have isomorphisms

$$
\left.\left.\left(R^{1} \Psi_{\mathcal{C}_{\bar{K}}} \Lambda\right)\right|_{x_{e}} \cong R^{1} \Psi_{W_{e}} \Lambda \cong\left(R^{1} \Psi_{\mathcal{X}} \Lambda\right)\right|_{x_{0}} \cong H^{1}\left(\mathbb{G}_{m, \bar{K}}, \Lambda\right)
$$

by [Be2, Theorem 3.1] and [SGA7II, Exposé XV Proposition 2.2.3]. Let $i_{0}$ and $i_{\infty}$ be the closed immersions of the zero point and the infinity point into $\mathbb{P}^{1}$ respectively. Then we have

$$
\begin{aligned}
& H^{1}\left(\mathbb{G}_{m, \bar{K}}, \Lambda\right) \cong H_{\mathrm{c}}^{1}\left(\mathbb{G}_{m, \bar{K}}, \Lambda\right)^{*}(-1), \\
& H_{\mathrm{c}}^{1}\left(\mathbb{G}_{m, \bar{K}}, \Lambda\right) \cong H^{0}\left(\mathbb{P}_{\bar{K}}^{1},\left(i_{0 *} \Lambda \oplus i_{\infty *} \Lambda\right) / \Lambda\right) .
\end{aligned}
$$

Hence the claim follows, because the zero point and the infinity point correspond to the irreducible components passing $x_{e}$.

We put

$$
H^{i}\left(\mathscr{W}_{\bar{k}}, R^{j} \Psi_{\mathscr{W}_{\mathcal{O}_{\mathbf{C}}}} \mathbb{Q}_{\ell}\right)=\left(\lim _{\stackrel{N}{\in \mathbb{N}}} H^{i}\left(\mathscr{W}_{\bar{k}}, R^{j} \Psi_{\mathscr{W}_{\mathcal{O}_{\mathbf{C}}}}\left(\mathbb{Z} / \ell^{N} \mathbb{Z}\right)\right)\right) \otimes_{\mathbb{Z}_{\ell}} \mathbb{Q}_{\ell}
$$

We consider $W_{\mathbf{C}}$ as a Berkovich space. Then we have a spectral sequence

$$
E_{2}^{i, j}=H^{i}\left(\mathscr{W}_{\bar{k}}, R^{j} \Psi_{\mathscr{W}_{\mathcal{O}_{\mathbf{C}}}} \mathbb{Q}_{\ell}\right) \Rightarrow H^{i+j}\left(W_{\mathbf{C}}, \mathbb{Q}_{\ell}\right)
$$

by [Fa, Proposition 5.9.4], because $W$ satisfies the condition in [Fa, Proposition 5.9.4] by Proposition 3.7. This spectral sequence gives an exact sequence

$$
0 \longrightarrow H^{1}\left(\mathscr{W}_{\bar{k}}, \mathbb{Q}_{\ell}\right) \longrightarrow H^{1}\left(W_{\mathbf{C}}, \mathbb{Q}_{\ell}\right) \longrightarrow H^{0}\left(\mathscr{W}_{\bar{k}}, R^{1} \Psi_{W_{\mathcal{O}_{\mathbf{C}}}} \mathbb{Q}_{\ell}\right) \longrightarrow H^{2}\left(\mathscr{W}_{\bar{k}}, \mathbb{Q}_{\ell}\right)
$$

by (4.1).

Let $\widetilde{\Gamma}_{\mathscr{W}}$ be the graph obtained by adding one new vertex to $\Gamma_{\mathscr{W}}$ and joining the new vertex with all vertices in $\mathcal{V}\left(\Gamma_{\mathscr{W}^{\prime}}\right) \backslash \mathcal{V}\left(\Gamma_{\mathscr{W}}\right)$. 
Lemma 4.2. There is a canonical isomorphism

$$
h: H_{1}\left(\widetilde{\Gamma}_{\mathscr{W}}, \mathbb{Q}_{\ell}\right)(-1) \stackrel{\sim}{\longrightarrow} \operatorname{Ker}\left(H^{0}\left(\mathscr{W}_{\bar{k}}, R^{1} \Psi_{\mathscr{W}_{\mathcal{O}_{\mathbf{C}}}} \mathbb{Q}_{\ell}\right) \longrightarrow H^{2}\left(\mathscr{W}_{\bar{k}}, \mathbb{Q}_{\ell}\right)\right) .
$$

Proof. By the isomorphism (4.2), we have a canonical isomorphism

$$
H^{0}\left(\mathscr{W}_{\bar{k}}, R^{1} \Psi_{\mathscr{W}_{\mathcal{O}_{\mathbf{C}}}} \mathbb{Q}_{\ell}\right) \cong H^{0}\left(\mathscr{W}_{\bar{k}},\left(\bigoplus_{v \in \mathcal{V}\left(\Gamma_{\mathscr{W}^{\prime}}\right)} \pi_{v *} \mathbb{Q}_{\ell}\right) / \mathbb{Q}_{\ell}\right)^{*}(-1) .
$$

Under the identification by (4.4), we explain the definition of $h$. For $e \in \mathcal{E}\left(\Gamma_{\mathscr{W}^{\prime}}\right)$, let $P_{e}$ be the point of $Y_{s(e)} \cap Y_{t(e)}$ that corresponds to $e$, and define $c_{e}$ as an element

$$
(0,1) \in\left(\left(\pi_{s(e)_{*}} \mathbb{Q}_{\ell} \oplus \pi_{t(e)_{*}} \mathbb{Q}_{\ell}\right) / \mathbb{Q}_{\ell}\right)_{P_{e}} \subset H^{0}\left(\mathscr{W}_{\bar{k}},\left(\bigoplus_{v \in \mathcal{V}\left(\Gamma_{\mathscr{W}^{\prime}}\right)} \pi_{v *} \mathbb{Q}_{\ell}\right) / \mathbb{Q}_{\ell}\right) .
$$

Then we define a $\mathbb{Q}_{\ell}$-linear map $h$ by

$$
h(r)\left(c_{e}\right)=\langle r, e\rangle
$$

for $r \in H_{1}\left(\widetilde{\Gamma}_{\mathscr{W}}, \mathbb{Q}_{\ell}\right) \subset E\left(\widetilde{\Gamma}_{\mathscr{W}}, \mathbb{Q}_{\ell}\right)$ and $e \in \mathcal{E}\left(\Gamma_{\mathscr{W}^{\prime}}\right)$, where $e$ is considered as an element of $E\left(\Gamma_{\mathscr{W}^{\prime}}, \mathbb{Q}_{\ell}\right) \subset E\left(\widetilde{\Gamma}_{\mathscr{W}}, \mathbb{Q}_{\ell}\right)$. Under the identification by (4.4), the last map in (4.3) is (-1)-twist of the dual of the natural map

$$
\bigoplus_{v \in \mathcal{V}\left(\Gamma_{\mathscr{W}}\right)} \mathbb{Q}_{\ell} \longrightarrow H^{0}\left(\mathscr{W}_{\bar{k}},\left(\bigoplus_{v \in \mathcal{V}\left(\Gamma_{W^{\prime}}\right)} \pi_{v *} \mathbb{Q}_{\ell}\right) / \mathbb{Q}_{\ell}\right) .
$$

Using this description, we can easily check that $h$ gives an isomorphism.

Proposition 4.3. We have two exact sequences

$$
\begin{aligned}
& 0 \longrightarrow H^{1}\left(\mathscr{W}_{\bar{k}}, \mathbb{Q}_{\ell}\right) \longrightarrow H^{1}\left(W_{\mathbf{C}}, \mathbb{Q}_{\ell}\right) \longrightarrow H_{1}\left(\widetilde{\Gamma}_{\mathscr{W}}, \mathbb{Q}_{\ell}\right)(-1) \longrightarrow 0 \\
& 0 \longrightarrow H^{1}\left(\Gamma_{\mathscr{W}}, \mathbb{Q}_{\ell}\right) \longrightarrow H^{1}\left(\mathscr{W}_{\bar{k}}, \mathbb{Q}_{\ell}\right) \longrightarrow \bigoplus_{v \in \mathcal{V}\left(\Gamma_{\mathscr{W}}\right)} H^{1}\left(\widetilde{Y}_{v}, \mathbb{Q}_{\ell}\right) \longrightarrow 0
\end{aligned}
$$

Proof. The first exact sequence follows from (4.3) and Lemma 4.2 ,

By a short exact sequence

$$
0 \longrightarrow \mathbb{Q}_{\ell} \longrightarrow \bigoplus_{v \in \mathcal{V}\left(\Gamma_{\mathscr{W}}\right)} \pi_{v *} \mathbb{Q}_{\ell} \longrightarrow\left(\bigoplus_{v \in \mathcal{V}\left(\Gamma_{\mathscr{W}}\right)} \pi_{v *} \mathbb{Q}_{\ell}\right) / \mathbb{Q}_{\ell} \longrightarrow 0
$$

on $\mathscr{W}_{\bar{k}}$, we have an exact sequence

$$
\begin{aligned}
\bigoplus_{v \in \mathcal{V}\left(\Gamma_{\mathscr{W}}\right)} H^{0}\left(\tilde{Y}_{v}, \mathbb{Q}_{\ell}\right) & \longrightarrow H^{0}\left(\mathscr{W}_{\bar{k}},\left(\bigoplus_{v \in \mathcal{V}\left(\Gamma_{\mathscr{W}}\right)} \pi_{v *} \mathbb{Q}_{\ell}\right) / \mathbb{Q}_{\ell}\right) \\
& \longrightarrow H^{1}\left(\mathscr{W}_{\bar{k}}, \mathbb{Q}_{\ell}\right) \longrightarrow \bigoplus_{v \in \mathcal{V}\left(\Gamma_{\mathscr{W}}\right)} H^{1}\left(\tilde{Y}_{v}, \mathbb{Q}_{\ell}\right) \longrightarrow 0
\end{aligned}
$$

This exact sequence and a canonical isomorphism

$$
\operatorname{Coker}\left(\bigoplus_{v \in \mathcal{V}\left(\Gamma_{\mathscr{W}}\right)} H^{0}\left(\tilde{Y}_{v}, \mathbb{Q}_{\ell}\right) \longrightarrow H^{0}\left(\mathscr{W}_{\bar{k}},\left(\bigoplus_{v \in \mathcal{V}\left(\Gamma_{\mathscr{W}}\right)} \pi_{v *} \mathbb{Q}_{\ell}\right) / \mathbb{Q}_{\ell}\right)\right) \cong H^{1}\left(\Gamma_{\mathscr{W}}, \mathbb{Q}_{\ell}\right)
$$

gives the second exact sequence.

Remark 4.4. Proposition 4.3 can be considered as an explicit description of a part of the weight spectral sequence for $W_{\mathbf{C}}(c f .[I l, 3.8])$. 


\section{Pushforward and pullback}

Let $W_{1}$ and $W_{2}$ be wide open rigid curves with semi-stable coverings $\mathcal{S}_{1}=\left\{\left(U_{1, i}, U_{1, i}^{\mathrm{u}}\right) \mid i \in I_{1}\right\}$ and $\mathcal{S}_{2}=\left\{\left(U_{2, i}, U_{2, i}^{\mathrm{u}}\right) \mid i \in I_{2}\right\}$ respectively. All the construction in the section 3 applies to $W_{1}$ and $W_{2}$, and the subscripts 1 and 2 mean that it is constructed from $W_{1}$ and $W_{2}$ respectively.

Definition 5.1. We say that a finite flat morphism $f: W_{1} \rightarrow W_{2}$ is compatible with semi-stable coverings if, for any $i_{1} \in I_{1}$, there is $i_{2} \in I_{2}$ such that $f\left(U_{1, i_{1}}^{\mathrm{u}}\right)=U_{2, i_{2}}^{\mathrm{u}}$ and $f\left(U_{1, i_{1}} \backslash U_{1, i_{1}}^{\mathrm{u}}\right)=$ $U_{2, i_{2}} \backslash U_{2, i_{2}}^{\mathrm{u}}$.

Let $f: W_{1} \rightarrow W_{2}$ be a finite flat morphism of degree $n$ that is compatible with semi-stable coverings. The morphism $f$ induces a finite morphism $\hat{f}: \mathscr{W}_{1} \rightarrow \mathscr{W}_{2}$ by Proposition 3.6. Further, $\hat{f}$ induces $\hat{f}_{\bar{k}}: \mathscr{W}_{1, \bar{k}} \rightarrow \mathscr{W}_{2, \bar{k}}$ and a finite flat morphism $\phi_{f}: \Gamma_{\mathscr{W}_{1}^{\prime}} \rightarrow \Gamma_{\mathscr{W}_{2}^{\prime}}$ of degree $n$. This induces a finite flat morphism $\phi_{f}: \Gamma_{\mathscr{W}_{1}} \rightarrow \Gamma_{\mathscr{W}_{2}}$ of degree $n$. The morphism $\phi_{f}$ naturally extends to a finite flat morphism $\widetilde{\phi}_{f}: \widetilde{\Gamma}_{\mathscr{W}_{1}} \rightarrow \widetilde{\Gamma}_{\mathscr{W}_{2}}$ of degree $n$.

In the remaining of this section, let $j=1,2$. We put $\mathscr{V}_{j}=\operatorname{Spf} \mathcal{O}_{K}\left[\left[S_{j}, T_{j}\right]\right] /\left(S_{j} T_{j}-c_{j}\right)$ for some $c_{j} \neq 0 \in \mathfrak{m}_{K}$. Let $Y_{j}$ and $Y_{j}^{\prime}$ be the closed subschemes of the geometric closed fiber $\mathscr{V}_{j, \bar{k}}$ defined by $T_{j}=0$ and $S_{j}=0$ respectively. We note that $Y_{j}=Y_{j}^{\prime}=\mathscr{V}_{j, \bar{k}}$. We put $\mathscr{V}_{j, \mathcal{O}_{\mathbf{C}}}=\mathscr{V}_{j} \widehat{\otimes}_{\mathcal{O}_{K}} \mathcal{O}_{\mathbf{C}}$ and

$$
H^{0}\left(\mathscr{V}_{j, \bar{k}}, R^{1} \Psi_{\mathscr{V}_{j, \mathcal{O}_{\mathbf{C}}}} \mathbb{Q}_{\ell}\right)=\left(\lim _{\overleftarrow{N \in \mathbb{N}}} H^{0}\left(\mathscr{V}_{j, \bar{k}}, R^{1} \Psi_{\mathscr{V}_{j, \mathcal{O}_{\mathbf{C}}}}\left(\mathbb{Z} / \ell^{N} \mathbb{Z}\right)\right)\right) \otimes_{\mathbb{Z}_{\ell}} \mathbb{Q}_{\ell}
$$

By (4.2) for $\mathscr{V}_{j}$, we have a canonical isomorphism

$$
H^{0}\left(\mathscr{V}_{j, \bar{k}}, R^{1} \Psi_{\mathscr{V}_{j, \mathcal{O}_{\mathbf{C}}}} \mathbb{Q}_{\ell}\right) \cong H^{0}\left(\mathscr{V}_{j, \bar{k}},\left(i_{Y_{j}} \mathbb{Q}_{\ell} \oplus i_{Y_{j *}^{\prime}} \mathbb{Q}_{\ell}\right) / \mathbb{Q}_{\ell}\right)^{*}(-1)
$$

where $i_{Y_{j}}: Y_{j} \rightarrow \mathscr{V}_{j, \bar{k}}$ and $i_{Y_{j}^{\prime}}: Y_{j}^{\prime} \rightarrow \mathscr{V}_{j, \bar{k}}$ are identity morphisms. We fix an identification $\mathbb{Q}_{\ell} \cong$

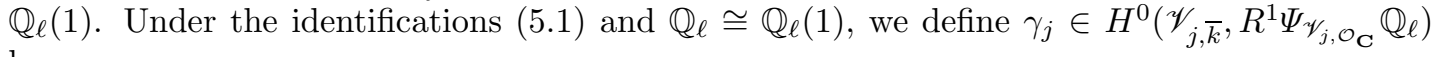
by

$$
\gamma_{j}\left(\left(a, a^{\prime}\right)\right)=a-a^{\prime} \quad \text { for } \quad\left(a, a^{\prime}\right) \in H^{0}\left(\mathscr{V}_{j, \bar{k}},\left(i_{Y_{j *}} \mathbb{Q}_{\ell} \oplus i_{Y_{j_{*}^{\prime}}} \mathbb{Q}_{\ell}\right) / \mathbb{Q}_{\ell}\right) .
$$

Lemma 5.2. Let $V_{j}$ be the open annulus associated to $\mathscr{V}_{j}$. Let $g: \mathscr{V}_{1} \rightarrow \mathscr{V}_{2}$ be a finite morphism such that the induced morphism $g: V_{1} \rightarrow V_{2}$ is a finite flat morphism of degree $m$. We assume that $g: \mathscr{V}_{1} \rightarrow \mathscr{V}_{2}$ induces a finite morphism $\operatorname{Spf} k\left[\left[S_{1}\right]\right] \rightarrow \operatorname{Spf} k\left[\left[S_{2}\right]\right]$. Let

$$
g_{*}: H^{0}\left(\mathscr{V}_{1, \bar{k}}, R^{1} \Psi_{\mathscr{V}_{1, \mathcal{O}_{\mathbf{C}}}} \mathbb{Q}_{\ell}\right) \longrightarrow H^{0}\left(\mathscr{V}_{2, \bar{k}}, R^{1} \Psi_{\mathscr{V}_{2, \mathcal{O}_{\mathbf{C}}}} \mathbb{Q}_{\ell}\right)
$$

be the pushforward by $g$, and let

$$
g^{*}: H^{0}\left(\mathscr{V}_{2, \bar{k}}, R^{1} \Psi_{\mathscr{V}_{2, \mathcal{O}_{\mathbf{C}}}} \mathbb{Q}_{\ell}\right) \longrightarrow H^{0}\left(\mathscr{V}_{1, \bar{k}}, R^{1} \Psi_{\mathscr{V}_{1, \mathcal{O}_{\mathbf{C}}}} \mathbb{Q}_{\ell}\right)
$$

be the pullback by $g$. Then we have $g_{*}\left(\gamma_{1}\right)=\gamma_{2}$ and $g^{*}\left(\gamma_{2}\right)=m \gamma_{1}$.

Proof. We have a canonical isomorphism

$$
H^{1}\left(V_{j, \mathbf{C}}, \mathbb{Q}_{\ell}\right) \cong H^{0}\left(\mathscr{V}_{j, \bar{k}}, R^{1} \Psi_{\mathscr{V}_{j, \mathcal{O}_{\mathbf{C}}}} \mathbb{Q}_{\ell}\right)
$$

by the similar exact sequence as (4.3) for $\mathscr{V}_{j}$. Hence, we may argue on the left hand side of (5.2). Let $N$ be a positive integer. Using the long exact sequence obtained from

$$
0 \longrightarrow\left(\mathbb{Z} / \ell^{N} \mathbb{Z}\right)(1) \longrightarrow \mathcal{O}_{V_{j, \mathbf{C}}}^{\times} \stackrel{\ell^{N}}{\longrightarrow} \mathcal{O}_{V_{j, \mathbf{C}}}^{\times} \longrightarrow 0,
$$


we have an injection $\mathcal{O}_{V_{j, \mathbf{C}}}^{\times} /\left(\mathcal{O}_{V_{j, \mathbf{C}}}^{\times}\right)^{\ell^{N}} \longrightarrow H^{1}\left(V_{j, \mathbf{C}},\left(\mathbb{Z} / \ell^{N} \mathbb{Z}\right)(1)\right)$. We consider a scheme $\mathcal{X}_{j}=$ $\operatorname{Spec} \mathcal{O}_{K}\left[S_{j}, T_{j}\right] /\left(S_{j} T_{j}-c_{j}\right)$. We consider a commutative diagram



where the right vertical arrow is an isomorphism by [Be2, Theorem 3.1]. From this commutative diagram, we obtain an isomorphism

$$
\mathcal{O}_{\mathcal{X}_{j, \bar{K}}}^{\times} /\left(\mathcal{O}_{\mathcal{X}_{j, \bar{K}}}^{\times}\right)^{\ell^{N}}(-1) \stackrel{\sim}{\rightarrow} H^{1}\left(V_{j, \mathbf{C}}, \mathbb{Z} / \ell^{N} \mathbb{Z}\right) .
$$

We show that $g^{*}$ induces

$$
\mathcal{O}_{\mathcal{X}_{2, \bar{K}}}^{\times} /\left(\mathcal{O}_{\mathcal{X}_{2, \bar{K}}}^{\times}\right)^{\ell^{N}} \rightarrow \mathcal{O}_{\mathcal{X}_{1, \bar{K}}}^{\times} /\left(\mathcal{O}_{\mathcal{X}_{1, K}}^{\times}\right)^{\ell^{N}} ; S_{2} \mapsto S_{1}^{m}
$$

We consider the natural isomorphism $V_{j} \cong A_{K}\left(\left|c_{j}\right|, 1\right)$ given by the parameter $S_{j}$. For $0<t<1$, we define a circle $C_{t}$ over $\mathbf{C}$ by

$$
C_{t}(\mathbf{C})=\{x \in \mathbf{C}|| x \mid=t\} .
$$

For $c \in \bar{K}$ such that $|c|<1$ and $|c|$ is sufficiently close to 1 , the morphism $g$ induces a finite flat morphism

$$
C_{|c|} \cong \operatorname{Sp} \mathbf{C}\left\langle X_{1}, X_{1}^{-1}\right\rangle \longrightarrow C_{m|c|} \cong \operatorname{Sp} \mathbf{C}\left\langle X_{2}, X_{2}^{-1}\right\rangle
$$

of degree $m$ such that $g^{*}\left(X_{2}\right)=c^{\prime} X_{1}^{m} g^{\prime}\left(X_{1}\right)$, where $X_{1}=S_{1} / c, X_{2}=S_{2} / c^{m}, c^{\prime} \neq 0 \in K(c)$ and

$$
g^{\prime}\left(X_{1}\right)=1+\sum_{k \neq 0} a_{k} X_{1}^{k} \in K(c)\left\langle X_{1}, X_{1}^{-1}\right\rangle
$$

for $a_{k} \in K(c)$ satisfying $\left|a_{k}\right|<1$. We take such $c \in \bar{K}$. Then $g^{\prime}\left(X_{1}\right)$ is $\ell$-divisible in $K(c)\left\langle X_{1}, X_{1}^{-1}\right\rangle$. This shows (5.4). Hence, we have that $g^{*}\left(\gamma_{2}\right)=m \gamma_{1}$ by (5.3). Then we have $g_{*}\left(\gamma_{1}\right)=\gamma_{2}$, because $g_{*} \circ g^{*}=m$ on $H^{1}\left(V_{2, \mathbf{C}}, \mathbb{Q}_{\ell}\right)$ by [Be, Theorem 5.4.1.(d)].

Theorem 5.3. Let $f: W_{1} \rightarrow W_{2}$ be a finite flat morphism that is compatible with semi-stable coverings. Then $f$ induces the following commutative diagrams:

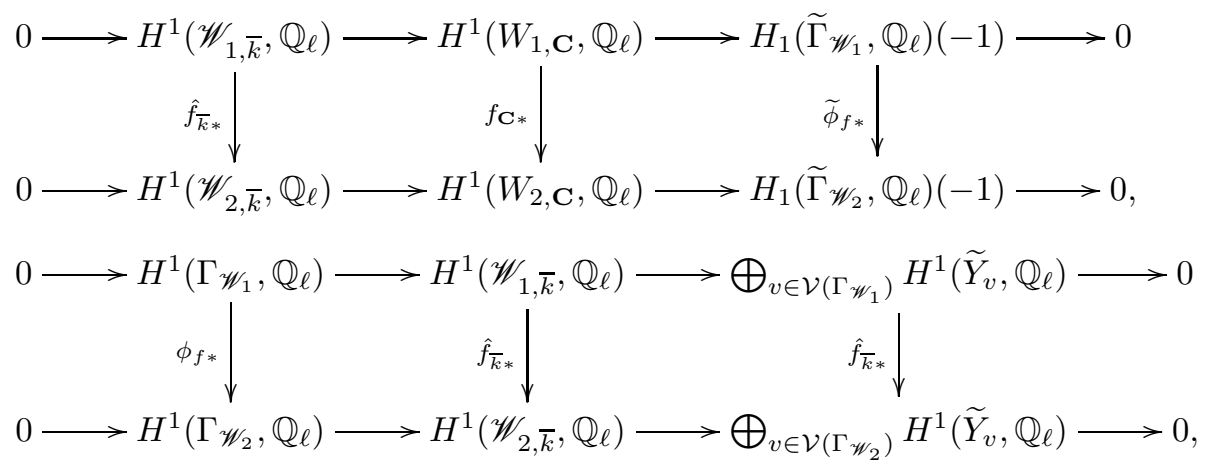




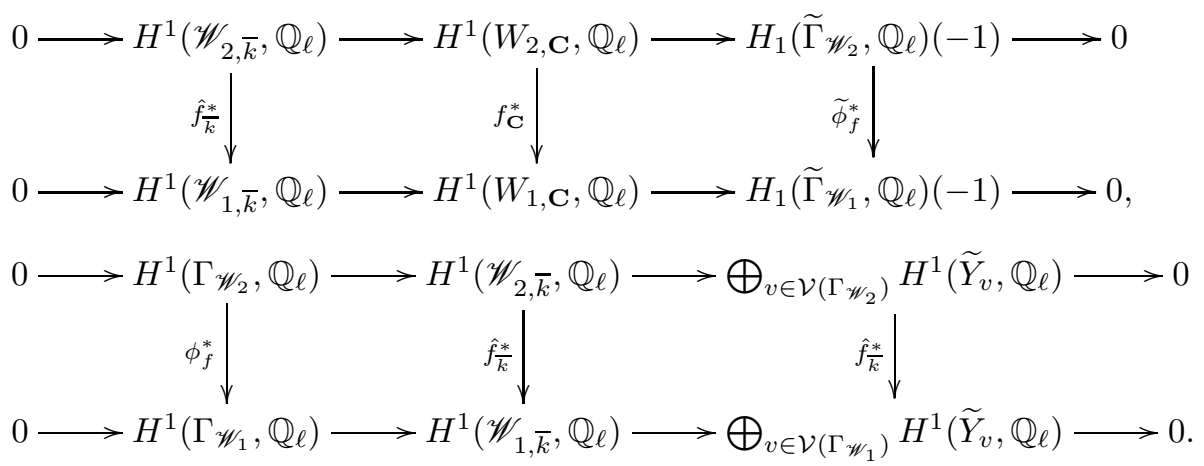

Proof. We can easily check the commutativities of the second and fourth diagrams from the construction of the short exact sequences. The commutativities of the former halves of the first and third diagrams are trivial.

For $e \in \mathcal{E}\left(\Gamma_{\mathscr{W}_{j}^{\prime}}\right)$, we define

$$
\begin{aligned}
\gamma_{e} \in & H^{0}\left(\mathscr{W}_{j, \bar{k}},\left(\left(\pi_{t(e)_{*}} \mathbb{Q}_{\ell} \oplus \pi_{s(e)_{*}} \mathbb{Q}_{\ell}\right) / \mathbb{Q}_{\ell}\right)^{*}(-1)\right) \\
& \subset H^{0}\left(\mathscr{W}_{j, \bar{k}},\left(\left(\bigoplus_{v \in \mathcal{V}\left(\Gamma_{\mathscr{W}_{j}^{\prime}}\right)} \pi_{v *} \mathbb{Q}_{\ell}\right) / \mathbb{Q}_{\ell}\right)^{*}(-1)\right)
\end{aligned}
$$

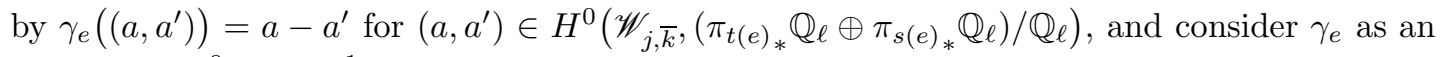
element of $H^{0}\left(\mathscr{W}_{j, \bar{k}}, R^{1} \Psi_{\mathscr{W}_{j, \mathcal{O}_{\mathbf{C}}}} \mathbb{Q}_{\ell}\right)$ by the canonical isomorphism

$$
R^{1} \Psi_{\mathscr{W}_{j, \mathcal{O}_{\mathbf{C}}}} \mathbb{Q}_{\ell} \cong\left(\left(\bigoplus_{v \in \mathcal{V}\left(\Gamma_{\mathscr{W}_{j}^{\prime}}\right)} \pi_{v *} \mathbb{Q}_{\ell}\right) / \mathbb{Q}_{\ell}\right)^{*}(-1)
$$

induced from (4.2) for $\mathscr{W}_{j}$. We define $\gamma_{e}=0$ for $e \in \mathcal{E}\left(\widetilde{\Gamma}_{\mathscr{W}_{j}}\right) \backslash \mathcal{E}\left(\Gamma_{\mathscr{W}_{j}^{\prime}}\right)$.

We show the commutativity of the latter half of the first diagram. We consider a cycle $R=e_{1} \cdots e_{m}$ of $\widetilde{\Gamma}_{\mathscr{W}_{1}}$ as an element of $H_{1}\left(\widetilde{\Gamma}_{\mathscr{W}_{1}}, \mathbb{Q}_{\ell}\right)$. Then it corresponds to $\sum_{i=1}^{m} \gamma_{e_{i}} \in$ $H^{0}\left(\mathscr{W}_{1, \bar{k}}, R^{1} \Psi_{\mathscr{W}_{1, \mathcal{O}_{\mathrm{C}}}} \mathbb{Q}_{\ell}\right)$. We have

$$
f_{*}\left(\sum_{i=1}^{m} \gamma_{e_{i}}\right)=\sum_{i=1}^{m} \gamma_{\widetilde{\phi}_{f, E}\left(e_{i}\right)} \in H^{0}\left(\mathscr{W}_{2, \bar{k}}, R^{1} \Psi_{\mathscr{W}_{2, \mathcal{O}_{\mathbf{C}}}} \mathbb{Q}_{\ell}\right)
$$

by Lemma 5.2, and this corresponds to $\widetilde{\phi}_{f *}(R)$.

We show the commutativity of the latter half of the third diagram. We consider a cycle $R^{\prime}=e_{1}^{\prime} \cdots e_{m}^{\prime}$ of $\widetilde{\Gamma}_{\mathscr{W}_{2}}$ as an element of $H_{1}\left(\widetilde{\Gamma}_{\mathscr{W}_{2}}, \mathbb{Q}_{\ell}\right)$. Then it corresponds to $\sum_{i=1}^{m} \gamma_{e_{i}^{\prime}} \in$ $H^{0}\left(\mathscr{W}_{2, \bar{k}}, R^{1} \Psi_{\mathscr{W}_{2, \mathcal{O}_{\mathbf{C}}}} \mathbb{Q}_{\ell}\right)$. We have

$$
f^{*}\left(\sum_{i=1}^{m} \gamma_{e_{i}^{\prime}}\right)=\sum_{i=1}^{m} \sum_{e \in \widetilde{\phi}_{f, E}^{-1}\left(e_{i}^{\prime}\right)} n_{e} \gamma_{e} \in H^{0}\left(\mathscr{W}_{1, \bar{k}}, R^{1} \Psi_{\mathscr{W}_{1, \mathcal{O}_{\mathbf{C}}}} \mathbb{Q}_{\ell}\right)
$$

by Lemma 5.2, and this corresponds to $\widetilde{\phi}_{f}^{*}\left(R^{\prime}\right)$. 


\section{References}

[Be] V. G. Berkovich, Étale cohomology for non-Archimedean analytic spaces, Inst. Hautes Études Sci. Publ. Math. 78 (1993), no. 1, 5-161.

[Be2] V. G. Berkovich, Vanishing cycles for formal schemes II, Invent. Math. 125 (1996), no. $2,367-390$.

[BGR] S. Bosch, U. Güntzer and R. Remmert, Non-Archimedean analysis, Grundlehren der Mathematischen Wissenschaften, 261, Springer-Verlag, Berlin, 1984.

[Co] R. Coleman, Reciprocity laws on curves, Compositio Math. 72 (1989), no. 2, 205-235.

[CM] R. Coleman and K. McMurdy, Stable reduction of $X_{0}\left(p^{3}\right)$, Algebra and Number Theory 4 (2010), no. 4, 357-431, with an appendix by Everette W. Howe.

[Fa] L. Fargues, Cohomologie des espaces de modules de groupes p-divisibles et correspondances de Langlands locales, Variétés de Shimura, espaces de Rapoport-Zink et correspondances de Langlands locales, Astérisque No. 291 (2004), 1-199.

[FvdP $] \quad$ J. Fresnel and M. van der Put, Rigid analytic geometry and its applications, Progress in Mathematics, 218, Birkhäuser Boston, Inc., Boston, MA, 2004.

[Il] L. Illusie, Autour du théorème de monodromie locale, Périodes p-adiques (Bures-surYvette, 1988), Astérisque No. 223 (1994), 9-57.

[deJ] A. J. de Jong, Crystalline Dieudonné module theory via formal and rigid geometry, Inst. Hautes Études Sci. Publ. Math. 82 (1995), no. 1, 5-96.

[Li] Q. Liu, Algebraic geometry and arithmetic curves, Oxford Graduate Texts in Mathematics, 6, Oxford Science Publications. Oxford University Press, Oxford, 2002.

[SGA7II] Groupes de monodromie en géometrie algébrique II, Séminaire de Géométrie Algébrique du Bois-Marie 1967-1969, dirigé par P. Deligne et N. Katz. Lecture Notes in Mathematics, Vol. 340, Springer-Verlag, Berlin-New York, 1973.

Naoki Imai

Graduate School of Mathematical Sciences, The University of Tokyo, 3-8-1 Komaba, Meguroku, Tokyo, 153-8914, Japan

naoki@ms.u-tokyo.ac.jp

Takahiro Tsushima

Graduate School of Mathematical Sciences, The University of Tokyo, 3-8-1 Komaba, Meguroku, Tokyo, 153-8914, Japan

tsushima@ms.u-tokyo.ac.jp 